\title{
Assessment of Transdermal Buprenorphine Patches for the Treatment of Chronic Pain in a UK Observational Study
}

\author{
Mick SerpelI $^{1} \cdot$ Shiva Tripathi $^{2} \cdot$ Sabine Scherzinger $^{3} \cdot$ Sònia Rojas-Farreras $^{4}$. \\ Alexander Oksche ${ }^{5,6} \cdot$ Margaret Wilson $^{7}$
}

Published online: 7 November 2015

(C) The Author(s) 2015. This article is published with open access at Springerlink.com

\begin{abstract}
Background Opioids provide effective analgesia for moderate-to-severe, chronic pain. Transdermal buprenorphine (TDB) is available in the UK as weekly, lower-dose $(5-20 \mu \mathrm{g} / \mathrm{h})$ patches and twice-weekly, higher dose $(35-70 \mu \mathrm{g} / \mathrm{h})$ patches. This prospective, observational, multicenter study of patients with various chronic pain conditions assessed the safety, perceptions, and discontinuation of treatment with TDB in a real-world, non-interventional setting (ClinicalTrials.gov study ID: NCT01225861).

Methods Patients aged $\geq 18$ years who were already receiving or initiating treatment with TDB were recruited in the UK during routine clinical visits and were followed for 6 visits or 9 months (whichever came first). Self-
\end{abstract}

Electronic supplementary material The online version of this article (doi:10.1007/s40271-015-0151-y) contains supplementary material, which is available to authorized users.

Margaret Wilson

maggie.wilson@mundipharma-rd.eu

1 Gartnavel General Hospital, University of Glasgow, Glasgow, UK

2 Royal Preston Hospital, Preston, UK

3 East Norwich Medical Partnership, Norwich, UK

4 IMS Health, Barcelona, Spain

5 Rudolf-Buchheim Institute of Pharmacology, Justus Liebig University Giessen (JLU Giessen), Giessen, Germany

6 Mundipharma Research GmbH \& Co. KG, Limburg, Germany

7 Mundipharma Research Ltd, Cambridge Science Park, Milton Road, Cambridge CB4 0GW, UK reported treatment adherence, patient satisfaction, and safety data were collected at each study visit.

Results Of 465 patients, 272 were already receiving 7-day TDB at the study start (TDB experienced), 146 were TDB naïve, and 47 were prescribed twice-weekly TDB. Most patients were female (72.9\%) and overweight/obese (body mass index $\geq 25: 75.3 \%$ ). The median age was 67 years, and the mean duration of pain was 11.1 years. Arthritis/other musculoskeletal disorders (39.6\%) were the most common causes of pain. Mild adverse events were commonly reported. Skin irritations, which were most frequent in 7-day TDB-experienced patients (45.6\%), rarely resulted in treatment discontinuation $(8.8 \%)$. Nearly all patients used TDB in accordance with treatment recommendations. Most patients reported that TDB was 'effective'/'very effective' at relieving pain and were 'satisfied'/'very satisfied' with TDB therapy.

Conclusion In everyday clinical practice, TDB was well tolerated and patients were satisfied with their therapy. Self-reported adherence to TDB was very high, and adverse events rarely resulted in treatment discontinuation. Opportunities were identified to limit common adverse events associated with TDB. 


\section{Key Points for Decision Makers}

Chronic pain is a highly debilitating condition commonly associated with physical and psychosocial impairments and a significant socioeconomic burden. Effective management often necessitates long-term treatment, which can be associated with suboptimal compliance and relapse.

This prospective, observational study indicates that real-world use of transdermal buprenorphine (TDB) in the UK is largely in accordance with the prescribing information.

Although many patients receiving TDB experienced at least one adverse event, these rarely resulted in treatment discontinuation. Patients also reported a high level of satisfaction with TDB therapy.

\section{Introduction}

Moderate-to-severe, chronic pain affects approximately one fifth of adults in Europe and is particularly prevalent in the elderly, being reported to affect over $70 \%$ of individuals aged $>65$ years $[1,2]$. It impairs patients' physical and psychological well-being and places a large financial burden on individuals and society [1,3,4]. Pharmacological treatments for chronic pain include traditional analgesics such as paracetamol and non-steroidal antiinflammatory drugs, with adjuvants such as antidepressants and anticonvulsants for neuropathic pain. The World Health Organization (WHO) analgesic ladder, developed for cancer-related pain, recommends a stepwise treatment approach, including step-2 weak opioid analgesics (e.g., codeine and tramadol) or more potent step-3 opioid analgesics (e.g., buprenorphine, morphine, and oxycodone) for selected patients with moderate-to-severe pain [5]. The evidence basis for managing non-cancer pain is less robust, with a recent meta-analysis reporting that paracetamol was ineffective for lower back pain and osteoarthritis [6]. Consequently, treatment guidelines for non-cancer pain generally support the WHO analgesic ladder [7, 8]. A large-scale survey of prescribing practices for opioid analgesics in the UK revealed that $83 \%$ of general practitioners considered opioids to be effective for chronic, non-malignant pain, although many had reservations about prescribing opioid analgesics long term [9]. While many patients benefit from prolonged-release oral opioids, transdermal formulations combine the convenience of a long duration of action with a simplified administration regimen and may particularly suit individuals wanting to reduce their pill count and those with swallowing difficulties or impaired gastrointestinal function $[10,11]$.

In vitro receptor-activation assays indicate that buprenorphine, along with fentanyl and morphine, acts as a partial agonist of the $\mu$-opioid receptor [12]. However, studies in healthy volunteers and heroin-dependent individuals reveal that oral buprenorphine at doses of $2 \mathrm{mg}$ occupies approximately $30-50 \%$ of $\mu$-opioid receptors in the central nervous system, while oral doses of $16 \mathrm{mg}$ and above occupy $79-95 \%$ of these receptors $[13,14]$. The slow dissociation of buprenorphine from $\mu$-opioid receptors results in a long duration of analgesic action, while antagonism of $\kappa$-opioid receptors exerts an antihyperalgesic effect [15-17]. Clinically relevant doses of buprenorphine have no analgesic ceiling effect, immunosuppressive activity, or effect on gonadal hormones, and pharmacokinetic properties are unaltered in elderly patients and individuals with renal dysfunction [10, 15, 16, 18]. Furthermore, the ceiling effects of buprenorphine on respiratory depression do not translate into a ceiling effect on analgesia [19].

Lower-dose (5-20 mg; nominal release rate 5-20 $\mu \mathrm{g} / \mathrm{h}$ ) transdermal buprenorphine (TDB) patches, administered weekly, are indicated for moderate, chronic, non-cancer pain, while higher dose (20-40 mg; nominal release rate 35-70 $\mu \mathrm{g} / \mathrm{h}$ ), twice-weekly TDB patches are indicated for patients with moderate-to-severe cancer pain or severe pain that does not respond to non-opioid analgesics [20, 21]. TDB can also be combined with short-acting opioid analgesics as a rescue medicine for breakthrough pain [22, 23]. A cross-sectional, UK study of opioid-prescribing patterns in primary care revealed a marked increase in the prescribing of buprenorphine between 2000 and 2010 [24].

In common with all opioid analgesics, buprenorphine has addictive properties and, as a Scheduled Drug, it is subject to stringent controls regarding its prescription, storage, and distribution. It is noteworthy that the pharmacokinetic properties of buprenorphine, including the ceiling effect on substance-induced euphoria, gradual systemic exposure, and low peak plasma concentrations for effectiveness, limit the 'likeability' of buprenorphine for abuse in comparison with other opioid analgesics [19]. In addition, the transdermal matrix patch renders buprenorphine particularly difficult to extract for illicit purposes [19].

Clinical trials demonstrate that TDB provides superior analgesia to placebo [25-29]. Furthermore, lower-dose TDB provides equivalent pain relief to sublingual buprenorphine and is non-inferior to prolonged-release tramadol or co-codamol [30-32]. Common events (occurring in $\geq 10 \%$ of patients) with TDB are typical of opioid analgesics and include constipation, dry mouth, nausea, 
vomiting, headache, dizziness, and somnolence [20, 21]. While skin irritations at the site of transdermal patch application are reported, information is lacking regarding the frequency, nature, and impact of skin irritations in a wider setting [20, 21, 33].

The nature of chronic pain generally necessitates longterm treatment, but many patients discontinue long-term opioid therapy because of adverse events (AEs), dosing schedules, and attitudes of others towards opioids [34]. We conducted a prospective, observational study to assess TDB in patients with chronic pain in a real-world, noninterventional setting. The primary objective was to establish the incidence and severity of AEs and reasons for discontinuing treatment with TDB in patients with chronic pain already treated with TDB and in those initiating therapy. Secondary aims were to gain insights into skin irritations associated with TDB, and patient and physician perceptions of treatment, and to assess self-reported adherence and satisfaction with TDB treatment in realworld clinical settings.

\section{Patients and Methods}

\subsection{Patients and Study Design}

Patients already receiving or initiating treatment with TDB were recruited into this single-arm, prospective, observational study (ClinicalTrials.gov study ID: NCT01225861) in the UK during routine clinical visits at 51 primary care (general practitioner) and ten secondary care (pain specialist) centers. Participating physicians were recruited from 12 Strategic Health Authorities (SHAs) distributed across the UK (South West, South Central, East of England, South East Coast, London, Scotland, Wales, East Midlands, Yorkshire and Humberside, North East, North West, West Midlands). Each SHA covered several Primary Care Trusts incorporating general practitioners and at least one pain clinic. Patients aged $<18$ years or with a life expectancy of $<6$ months were excluded from the study. The first patient first visit and last patient last visit occurred in January 2011 and February 2014, respectively. The study comprised a baseline recruitment visit and six observational (follow-up) visits (or 9 months on-study, whichever came first), which were conducted during routine clinical consultations (Fig. 1).

The study was performed in accordance with the regulations and guidelines governing medical practice and ethics in the UK, following National Research Ethics Service approval. Local approval was obtained from the Research and Development Departments of the participating secondary care centers. For each primary care site, the responsible Clinical Commissioning Group or Primary
Care Trust was notified and approval was obtained as necessary. All study documents were reviewed and approved by the appointed Research Ethics Committee, and all patients provided informed, written consent. No patient-identifiable data were collected.

\subsection{Outcomes and Assessments}

Data on AEs with TDB, self-reported treatment adherence, patient satisfaction, and perceptions of treatment were collected at regular intervals throughout the study via questionnaires. Patient demographic data and medical history were also assessed.

Patient Questionnaire A (completed at baseline) and Patient Questionnaire B (completed at each follow-up visit) evaluated the effectiveness, application/self-reported adherence, and satisfaction with 7-day TDB; various parameters of the Brief Pain Inventory assessment tool; and AEs, including skin irritations. Patient Questionnaire A also evaluated the medical history, prior treatments for pain, and personal circumstances/general well-being. Treatment switches (including reasons for switching) were captured in Patient Questionnaire B.

Physician Questionnaire A (completed at baseline) and Physician Questionnaire B (completed at each follow-up visit) evaluated the effectiveness of 7-day TDB; AEs, including skin irritations and serious AEs; and treatment switches (including the reasons for switching). Physician Questionnaire A also evaluated the medical history, comorbidities, prior treatments for pain, and satisfaction with 7-day TDB (See the Electronic Supplementary Material for further details of the Patient and Physician Questionnaires).

A separate Study Discontinuation Form was also completed if the patient discontinued TDB therapy, to evaluate the reasons for discontinuation, including the characteristics of AEs, the duration of TDB therapy, and follow-up treatments (Fig. 1).

\subsection{Statistical Methods}

The analyses were based on data collected in the Patient/ Physician Questionnaires. Outcomes are described for all patients meeting the study inclusion criteria. Patients were stratified according to the type of TDB product: 7-day TDB [5 mg, $10 \mathrm{mg}$, or $20 \mathrm{mg}$ patch strengths (equivalent to $5-20 \mu \mathrm{g} / \mathrm{h}$ )] or twice-weekly TDB [20 mg, $30 \mathrm{mg}$, or $40 \mathrm{mg}$ patch strengths (equivalent to $35-70 \mu \mathrm{g} / \mathrm{h}$ )]. The 7-day TDB population was further stratified as treatment experienced (patients receiving 7-day TDB at study enrolment for $>30$ days) or treatment naïve (patients who had not received 7-day TDB prior to study onset for $>30$ days). Further analyses were conducted in patients 


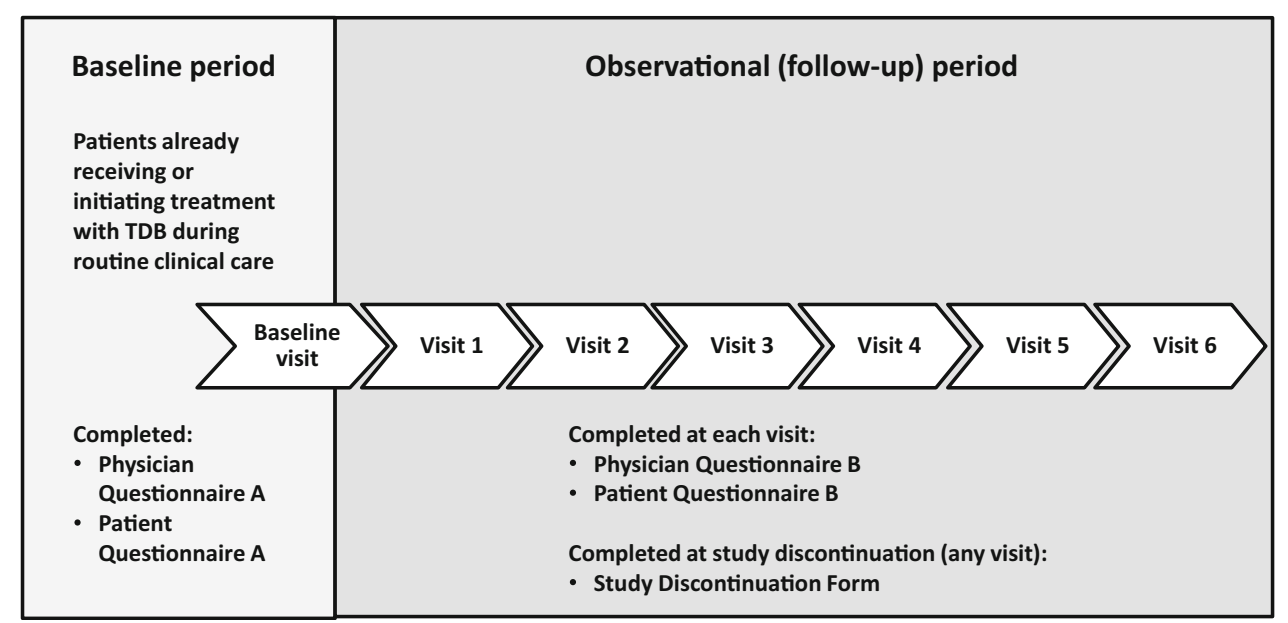

Fig. 1 The study comprised a baseline period [during which patients already receiving or initiating treatment with transdermal buprenorphine (TDB) were recruited during routine clinical care, and Physician Questionnaire A and Patient Questionnaire A were completed] and an observational (follow-up period) of 9 months or six follow-up visits (whichever came first). At each study visit, Physician Questionnaire B and Patient Questionnaire B were completed. A Study Discontinuation Form was completed at study discontinuation

$>30$ days and $n=146(34.9 \%)$ were TDB naïve. More patients were recruited by primary care physicians $(n=381)$ than by pain specialists $(n=84)$. The mean [standard deviation (SD)] durations of follow-up were 3.3 (3.5) months for all patients (range 0.0-16.7 months) and 5.0 (3.3) months for those with $\geq 1$ follow-up visit ( $n=304$, range $0.2-16.7$ months).

Demographic and disease characteristics were comparable for patients receiving 7-day and twice-weekly TDB (Table 1). Overall, most patients were female, of white ethnicity, and either overweight or obese. Patients had been receiving analgesic medication for a mean (SD) of 10.0 (10.0) years. The most common primary pain diagnosis was arthritis or another musculoskeletal inflammatory disorder, and the most common comorbidities were depression, constipation, drug hypersensitivity, and asthma $(n=91,19.6 \%)$. Constipation at baseline was most frequent in the twice-weekly TDB cohort $(n=17,36.2 \%)$ compared with TDB-experienced patients $(n=80$, $29.4 \%)$ and TDB-naïve patients $(n=32,21.9 \%)$. Other atopic allergic comorbidities included hay fever $(n=25$, $5.4 \%)$, rash or hives $(n=17,3.7 \%)$, and contact allergies $(n=15,3.2 \%)$. The main reasons for initiating TDB treatment were to reduce the number of oral medications $(32.0 \%)$, pain control (31.9\%), compliance $(20.4 \%)$, and failure on other analgesics $(20.3 \%)$.

Twice-weekly TDB was prescribed at a higher mean (SD) dose [30.2 (12.1) mg] than 7-day TDB. Patients who were 7-day TDB experienced were prescribed a higher mean (SD) dose of TDB than TDB-naïve patients [12.5 (6.8) $\mathrm{mg}$ versus $7.2(4.1) \mathrm{mg}$. Seven-day TDB $5 \mathrm{mg}$ patches were more commonly prescribed for treatment-naïve 
Table 1 Patient demographic and disease characteristics at baseline

\begin{tabular}{|c|c|c|}
\hline Characteristic & 7 -day TDB $(n=418)$ & $\begin{array}{l}\text { Twice-weekly } \\
\text { TDB }(n=47)\end{array}$ \\
\hline Median age, years (range) & $68(22-99)$ & $61(31-96)$ \\
\hline Female, $n(\%)$ & $307(73.4)$ & $32(68.1)$ \\
\hline Body mass index, mean (SD) & $29.4(6.7)$ & $29.3(6.0)$ \\
\hline \multicolumn{3}{|l|}{ Primary diagnosis, $n(\%)$} \\
\hline Arthritis or musculoskeletal inflammatory disease & $172(41.1)$ & $12(25.5)$ \\
\hline Spinal/back pain condition or injury & $147(35.2)$ & $13(27.7)$ \\
\hline Musculoskeletal pain or injury & $50(12.0)$ & $9(19.1)$ \\
\hline Bone disease and related pain & $19(4.5)$ & $2(4.3)$ \\
\hline Neurological disorder & $17(4.1)$ & $3(6.4)$ \\
\hline Other $^{\mathrm{a}}$ & $13(3.1)$ & $8(17.2)$ \\
\hline Mean duration of chronic pain, years (SD) & $11.2(10.8)$ & $10.7(7.3)$ \\
\hline Mean pain score on numerical analog scale ${ }^{\mathrm{b}}$ during previous 7 days (SD) & $6.0(2.1)$ & $6.1(2.3)$ \\
\hline Mean duration of treatment for chronic pain, years (SD) & $10.0(10.3)$ & $9.6(7.3)$ \\
\hline \multicolumn{3}{|l|}{ Common concomitant morbidities ${ }^{\mathrm{c}}, n(\%)$} \\
\hline Depression & $131(31.3)$ & $14(29.8)$ \\
\hline Constipation & $112(26.8)$ & $17(36.2)$ \\
\hline Drug hypersensitivity & $111(26.6)$ & $8(17.0)$ \\
\hline Asthma & $81(19.4)$ & $10(21.3)$ \\
\hline Autoimmune or chronic inflammatory disorder & $47(11.2)$ & $6(12.8)$ \\
\hline Gait and balance disorder & $44(10.5)$ & $6(12.8)$ \\
\hline
\end{tabular}

$S D$ standard deviation, $T D B$ transdermal buprenorphine

${ }^{a}$ Other primary diagnoses included cancer $(1.7 \%)$, inflammatory diseases $(1.1 \%)$, dermatological conditions $(1.1 \%)$, renal and genitourinary disorders $(0.4 \%)$, and lymph/circulatory disorders $(0.2 \%)$

${ }^{\mathrm{b}}$ Numerical analog scale (from $0=$ no pain to $10=$ worst pain imaginable)

c Common concomitant morbidities occurring in $\geq 10 \%$ of the total study population [other atopic allergic comorbidities included hay fever $(n=25,5.4 \%)$, rash or hives $(n=17,3.7 \%)$, and contact allergies $(n=15,3.2 \%)$ ]

patients than for treatment-experienced patients $(70.5$ versus $22.4 \%$ ), while fewer treatment-naive patients than treatment-experienced patients received $10 \mathrm{mg}$ TDB patches (22.6 versus $42.6 \%)$. Most patients $(81.3 \%)$ received concomitant analgesic medication in parallel with TDB during the study. The most frequently coprescribed analgesics were paracetamol $(46.0 \%)$, paracetamol combinations excluding psycholeptics (13.1\%), and tramadol $(11.8 \%)$. Amitriptyline was coprescribed in $13.1 \%$ of patients. $^{1}$

Overall, $80.9 \%$ of the 465 patients continued treatment with TDB during the follow-up period. The discontinuation rate was higher in TDB-naïve patients $(34.2 \%)$ than in TDB-experienced patients $(12.1 \%)$ and those receiving twice-weekly TBD (12.8\%). In the overall population, the most common reason for discontinuing treatment was AEs $(n=56,12.0 \%$; Fig. 2). Very few patients discontinued

\footnotetext{
${ }^{1}$ Amitriptyline may have been prescribed for analgesia, depression, or both conditions $(31.2 \%$ of patients had depression as a comorbidity).
}

treatment because of self-reported lack of adherence (i.e., the patient stopped using TDB: $n=3,0.6 \%$ ). At treatment discontinuation, the mean (SD) twice-weekly and 7-day TDB doses were 68.1 (24.3) $\mathrm{mg}$ and 11.4 (9.5) mg, respectively, and 7-day TDB-experienced patients were receiving a numerically lower mean $(\mathrm{SD})$ patch strength [9.7 (5.8) $\mathrm{mg}$ ] than TDB-naïve patients [12.5 (11.2) $\mathrm{mg}$, $p=0.564]$.

\subsection{Safety: Excluding Skin Irritations}

AEs, excluding skin irritations, were experienced by $50.1 \%$ of patients, the most common events being constipation $(28.0 \%)$, nausea (16.6\%), dizziness $(10.3 \%)$, sleeping disorder $(10.1 \%)$, and vomiting $(3.9 \%)$. The incidence rates per 100 patient-years were $20.6,12.9,7.7$, 7.4, and 3.5 for constipation, nausea, dizziness, sleeping disorder, and vomiting, respectively.

A higher proportion of 7-day TDB-experienced patients than TDB-naive patients reported constipation (30.9 versus $20.5 \%, p=0.0237$ ), which also persisted for longer in 
Fig. 2 The reasons for discontinuing treatment with transdermal buprenorphine in the overall population $(n=465)$ were adverse events $(12.0 \%)$, lack of effectiveness (4.1\%), other reasons (3.9\%), and lack of self-reported adherence $(0.6 \%)$

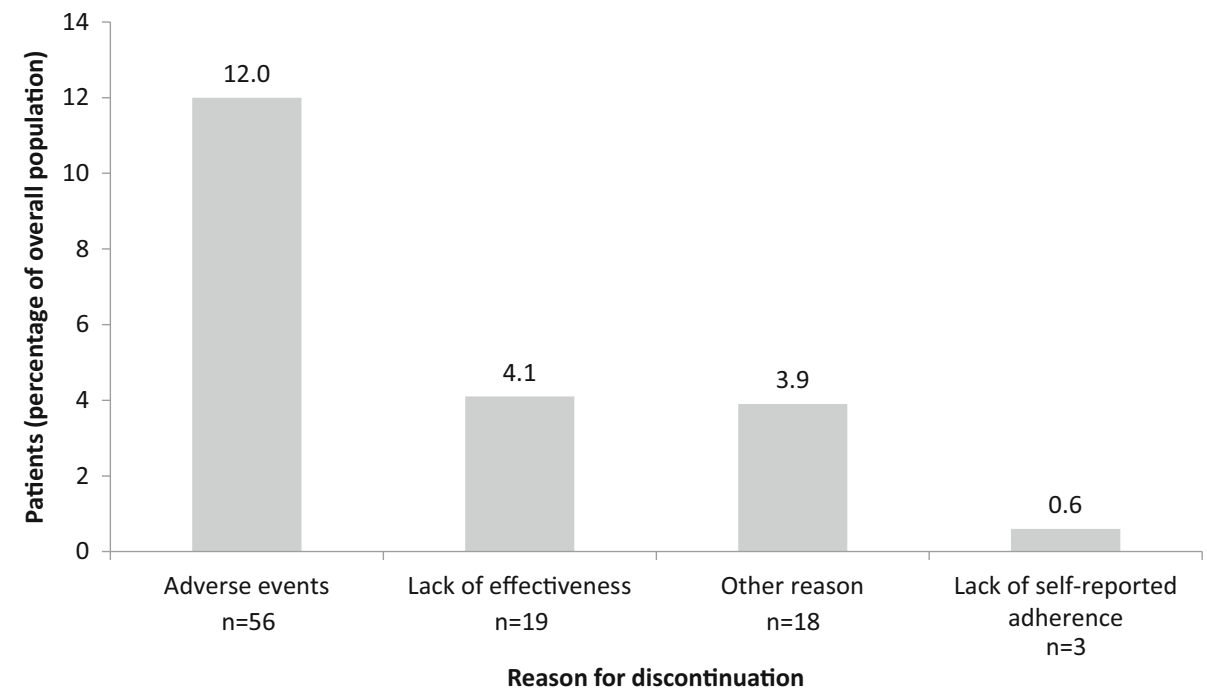

treatment-experienced patients [mean (SD) 233.7 (361.1) versus 50.5 (49.2) days, $p=0.0154]$. Constipation was generally rated as moderate $(47.7 \%)$ or mild $(33.3 \%)$. Dizziness was less frequent in TDB-experienced patients than in TDB-naïve patients (7.4 versus $17.1 \%$, $p=0.0021)$, but, when present, it persisted for longer in TDB-experienced patients [mean (SD) 168.4 (363.7) versus 15.6 (17.9) days, $p=0.0192]$. Nausea, vomiting, and sleep disturbance were experienced by similar proportions of treatment-experienced and treatment-naïve patients. Nausea and dizziness were most frequently rated as mild (46.6 and $45.5 \%$, respectively), sleep disturbance was most frequently rated as moderate $(72.0 \%)$, and vomiting was most frequently rated as severe $(56.0 \%)$. Concomitant medications were received by $68.4,50.0,40.8,24.4$, and $15.6 \%$ of patients experiencing constipation, vomiting, nausea, sleep disorders, and dizziness, respectively, to manage these events.

\subsection{Safety: Skin Irritations}

Skin irritation was the most frequently reported AE, being more common in 7-day TDB-experienced patients $(45.6 \%)$ than in treatment-naïve patients $(32.9 \%)$. However, very few patients experiencing skin irritation discontinued TDB therapy because of the skin irritation (8.8\%, Fig. 3). Overall, $41.1 \%$ of patients receiving TDB experienced an average of 1.3 skin irritations. Skin irritations (Table 2 ) were generally short lasting $(80.6 \%$ ), mild $(53.3 \%)$, or moderate $(33.0 \%)$ in severity and were described as an itching sensation $(60.7 \%)$ or a burning sensation $(18.8 \%)$. Almost all skin irritations were limited to within the patch area (98.4\%), and TDB therapy was suspected to be the cause of skin irritation in $97.0 \%$ of cases. The 7-day TDB-experienced group with skin irritation experienced significantly more erythema than the TDB-naïve group (76.6 versus $54.2 \%, p=0.0038$ ) and had numerically fewer patients with 'no evidence of skin irritation' (21.8 versus $35.4 \%, p=0.0659)$. Overall, $25.7 \%$ of patients experiencing skin irritation received treatment. Hydrocortisone was most commonly used by patients receiving 7-day TDB, while emollients/protectives were used most frequently by patients receiving twiceweekly TDB. Most patients who received treatment for skin reaction considered it to be at least 'somewhat effective' (77.6 \%).

Analyses performed only in 7-day TDB patients revealed that skin irritations tended to be more common in patients who reported a history of certain allergies, including skin reactions to food (no skin reaction $2.2 \%$ versus $\geq 1$ skin reaction $15.1 \%, p=0.0028)$ and skin reactions to perfumes, cosmetics, and washing powder (15.7 versus $37.0 \%, p=0.002$ ). There was no significant difference in the incidence of skin irritations according to patient-reported asthma, hay fever, or skin reaction to pets. However, the physician-reported data showed that skin irritations were more common (no skin reaction versus $\geq 1$ skin reaction) in patients with hay fever (3.3 versus $8.7 \%$, $p=0.0158$ ). A numerically greater incidence of skin irritations was also seen in patients with physician-reported drug hypersensitivity (23.6 versus $30.8 \%, p=0.0992$ ) or psoriasis (3.3 versus $7.0 \%, p=0.0791$ ), while skin irritations were less common in patients with autoimmune/ chronic inflammatory disorders (13.8 versus $7.6 \%$, $p=0.0461$ ).

Approximately half of the patients $(45.8 \%)$ who experienced skin irritation reported that they took no action. Of the actions taken, the most frequent was to remove the TDB patch and apply a new patch to a different skin location (32.9\%; Fig. 3). 


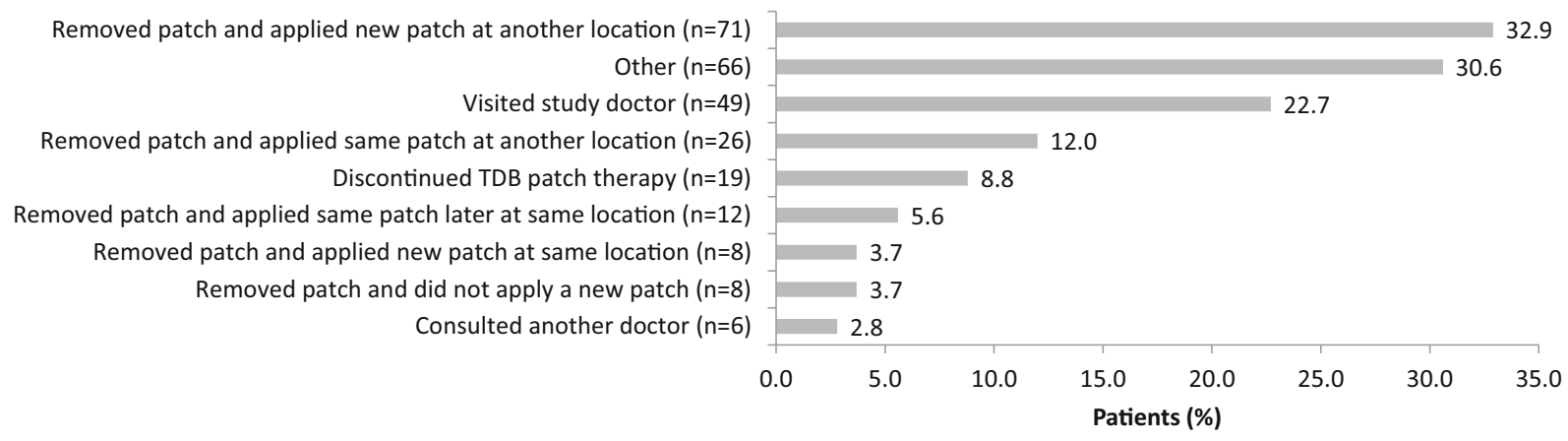

Fig. 3 The most common patient-reported actions $(n=216)$ with transdermal buprenorphine (TDB) patches in response to skin irritation (a multiresponse question) were 'removed patch and applied a new patch to another location' (32.9\%), 'other' response (including

Some events of skin irritations reported by physicians were not reported by patients: 48 of 222 and 10 of 25 physician-reported skin irritation AEs were not reported by patients in the 7-day TDB and twice-weekly TDB groups, respectively.

\subsection{Self-Reported Adherence and Treatment Satisfaction}

Seven-day TDB-naïve and TDB-experienced patients wore patches for a median of 7 days (95\% CI 6.7-6.9), and twiceweekly TDB patients wore patches for a median of 3.5 days (95\% CI 3.5-4.3). Patients had applied patches to a median of four different skin locations in the previous 4 weeks. Almost all patients did not remove and reapply patches during the dosing interval $(97.2 \%)$ or cut the patches $(98.5 \%)$. During the previous 4 weeks, $3.1 \%$ of patients said they had applied more patches than they were prescribed and $6.4 \%$ said they had forgotten to apply a patch. There were no significant differences in measures of self-reported adherence for 7-day TDB-naïve versus TDB-experienced patients.

Treatment satisfaction data are available for a longer length of time than the study observation period, as some patients were receiving TDB therapy prior to study enrolment. Most patients reported that 7-day TDB was 'effective' or 'very effective' in relieving their pain (Fig. 4a). Patients were more likely to find 7-day TDB 'not very effective' during the first 3 months of therapy (approximately one third), and 'effective'/'very effective'with increasing time on therapy. Most patients 'fully agreed' or 'agreed' that their skin tolerated TDB patches well across the entire study period [from $55.6 \%$ (in month 15) to $87.5 \%$ (in month 36 ); Fig. 4b]. Most patients were also satisfied overall with TDB therapy; satisfaction rates remained above $70 \%$ for the entire treatment duration assessed (Fig. 4c). Treatment satisfaction was also high in patients experiencing at least one skin irritation: $62.9 \%$ (in month 15 ) to $93.5 \%$ (in month $>36$ ) of this patient group were satisfied overall with 'cream applied', 'kept patch on, no action', 'reduced dosage', 'scratch', 'took antihistamines', and 'washed and dried site'; $30.6 \%)$, and 'visited study doctor' $(22.7 \%)$

TDB. More than $65 \%$ of patients also favored TDB above their previous analgesic medication throughout the followup period. There were no consistent differences in treatment satisfaction parameters between treatment-naïve and treatment-experienced patients.

\section{Discussion}

This observational, prospective, multicenter study describes the AEs, reasons for treatment discontinuation, satisfaction, self-reported adherence, and characteristics of 465 patients receiving TDB in the UK healthcare system. Since the study sites were well distributed throughout the UK, these findings are likely to be representative of the wider UK population.

This study suggests that UK patients are receiving TDB in accordance with the prescribing information [20, 21]. For example, patients were experiencing chronic pain (average duration 11 years), which was predominantly non-cancer in origin and of moderate-to-severe intensity. The patients receiving TDB in this study largely reflected the wider UK population with chronic pain [35]. Most patients were older (median age 67 years), and over half had a primary pain diagnosis of arthritis or a musculoskeletal inflammatory disease, or a spinal/back pain condition or injury. Physician-reported baseline allergic conditions were common in patients in this study and included drug sensitivities (25.6\%) and asthma (19.6\%). The observation that most patients $(81.3 \%)$ prescribed TDB were also receiving concomitant analgesic medications is in line with the WHO stepwise analgesic ladder [5]. However, because of the non-interventional design of this study, we cannot ascertain the impact of these concomitant medications on the AEs observed.

Skin irritation was the most frequently reported $\mathrm{AE}$ $(41.1 \%)$ reported with TDB, followed by constipation $(28.0 \%)$ and nausea $(16.6 \%)$. The high incidence of 
Table 2 Skin irritations occurring during treatment with transdermal buprenorphine (TDB): patients with $\geq 1$ skin irritation during follow-up ${ }^{\mathrm{a}}$

\begin{tabular}{|c|c|c|c|}
\hline Parameter & 7-day TDB experienced & 7-day TDB naïve & Twice-weekly TDB \\
\hline \multicolumn{4}{|l|}{ Intensity of skin irritation, $n(\%)^{\mathrm{b}}$} \\
\hline No evidence of irritation & $27(21.8)$ & $17(35.4)$ & $5(26.3)$ \\
\hline Erythema & $95(76.6)$ & $26(54.2)$ & $17(89.5)$ \\
\hline Erythema and papules & $25(20.2)$ & $13(27.1)$ & $7(36.8)$ \\
\hline Erythema, papules and vesicle & $6(4.8)$ & $3(6.3)$ & $1(5.3)$ \\
\hline Strong reaction spreading beyond test site & 0 & $3(6.3)$ & 0 \\
\hline Median duration of skin irritation, days (range) ${ }^{c}$ & $94(1-2481)$ & $23(1-405)$ & $167(1-1716)$ \\
\hline \multicolumn{4}{|l|}{ Severity of skin reaction, $n(\%)^{\mathrm{d}}$} \\
\hline Mild & $74(56.9)$ & $27(49.1)$ & $12(44.4)$ \\
\hline Moderate & $40(30.8)$ & $17(30.9)$ & $13(48.1)$ \\
\hline Severe & $16(12.3)$ & $11(20.0)$ & $2(7.4)$ \\
\hline \multicolumn{4}{|l|}{ Progression of skin reaction, $n(\%)^{\mathrm{b}}$} \\
\hline Short lasting & $104(83.9)$ & $33(68.8)$ & $17(89.5)$ \\
\hline Long lasting & $23(18.5)$ & $12(25.0)$ & $3(15.8)$ \\
\hline Long lasting and worsening over time & $4(3.2)$ & $4(8.3)$ & 0 \\
\hline Skin reaction suspected to be treatment related, $n(\%)^{\mathrm{e}}$ & $139(95.9)$ & $57(100.0)$ & $27(96.4)$ \\
\hline \multicolumn{4}{|l|}{ Pathogenic nature of skin reaction, $n(\%)^{\mathrm{b}}$} \\
\hline Allergic & $22(17.7)$ & $12(25.0)$ & $5(26.3)$ \\
\hline Irritant & $69(55.6)$ & $21(43.8)$ & $12(63.2)$ \\
\hline Toxic & $11(8.9)$ & $3(6.3)$ & $1(5.3)$ \\
\hline Infectious & 0 & $1(2.1)$ & 0 \\
\hline Unclear & $26(21.0)$ & $10(20.8)$ & $3(15.8)$ \\
\hline Received treatment for skin irritation, $n(\%)^{\mathrm{b}}$ & $28(22.6)$ & $14(29.2)$ & $7(36.8)$ \\
\hline \multicolumn{4}{|l|}{ Treatment received for skin irritation, $n(\%)^{\mathrm{f}}$} \\
\hline Emollient and protectives & $7(25.0)$ & $4(28.6)$ & $7(100.0)$ \\
\hline Hydrocortisone & $16(57.1)$ & $9(64.3)$ & 0 \\
\hline Betamethasone & $1(3.6)$ & 0 & $1(14.2)$ \\
\hline Clobetasol propionate & $3(10.7)$ & 0 & 0 \\
\hline Beclomethasone & $3(10.7)$ & 0 & 0 \\
\hline Fexofenadine & 0 & $3(21.4)$ & 0 \\
\hline \multicolumn{4}{|l|}{ Effectiveness of treatment for skin irritation, $n(\%)^{\mathrm{f}}$} \\
\hline Completely effective & $8(28.6)$ & $5(35.7)$ & $2(28.6)$ \\
\hline Very effective & $4(14.3)$ & $2(14.3)$ & $1(14.3)$ \\
\hline Somewhat effective & $10(35.7)$ & $4(28.6)$ & $2(28.6)$ \\
\hline Not very effective & $2(7.1)$ & $1(7.1)$ & $1(14.3)$ \\
\hline Not at all effective & $3(10.7)$ & $2(14.3)$ & 0 \\
\hline
\end{tabular}

a 124 7-day TDB-experienced patients (45.6\%), 48 7-day TDB-naïve patients (32.3\%), and 19 twice-weekly TDB patients (40.4 \%) experienced $\geq 1$ skin irritation during follow-up

b Assessed in 124, 48, and 19 7-day TDB-experienced, 7-day TDB-naïve, and twice-weekly TDB patients, respectively (progression of skin reaction was a multiresponse question)

c Assessed in 97, 44, and 20 7-day TDB-experienced, 7-day TDB-naïve, and twice-weekly TDB patients, respectively

d Assessed in 130, 55, and 27 cases of skin irritation in 7-day TDB-experienced, 7-day TDB-naïve, and twice-weekly TDB patients, respectively

e Assessed in 145, 57, and 28 cases of skin irritation in 7-day TDB-experienced, 7-day TDB-naïve, and twice-weekly TDB patients, respectively (patient-reported, multiresponse question)

${ }^{\mathrm{f}}$ Assessed in 28, 14, and seven 7-day TDB-experienced, 7-day TDB-naïve, and twice-weekly TDB patients, respectively (percentages calculated with the number of patients prescribed treatment for skin irritation as the denominator; type of treatment was a patient-reported, multiresponse question; data on treatment effectiveness are missing for one 7-day TDB-experienced patient and one twice-weekly TDB patient) 
Fig. 4 Patient-reported satisfaction with transdermal buprenorphine patches (in all patients with available data). a Overall treatment effectiveness (only in patients receiving 7-day TDB). b Skin tolerability of treatment. c Overall satisfaction with treatment

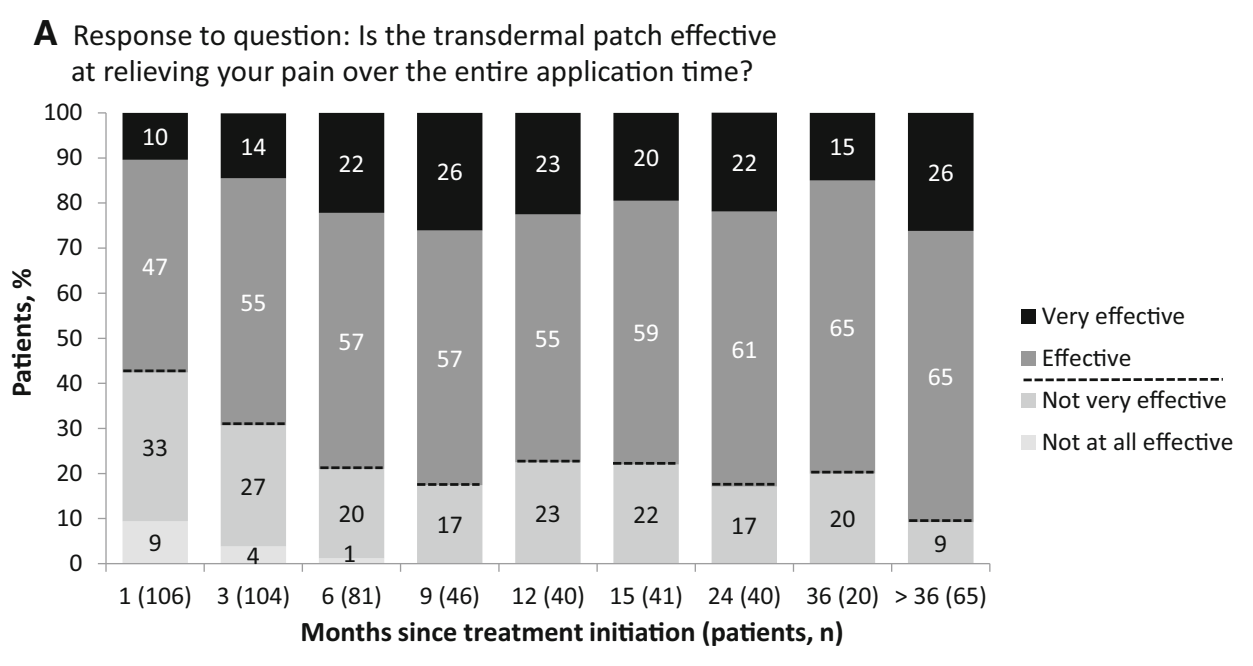

B Response to statement: My skin tolerates the patch well

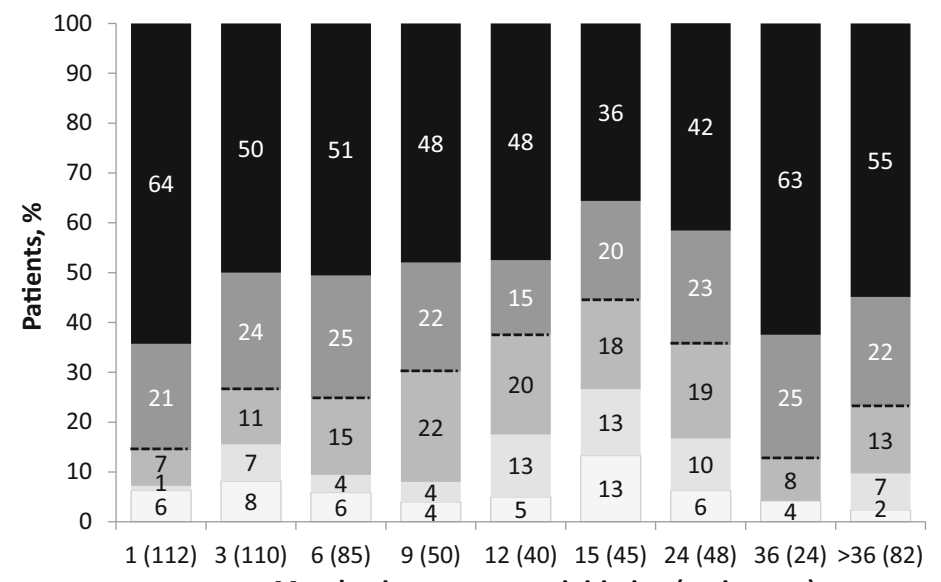

- 5. fully agree

4. agree

3. neither agree or disagree

2. disagree

1. strongly disagree

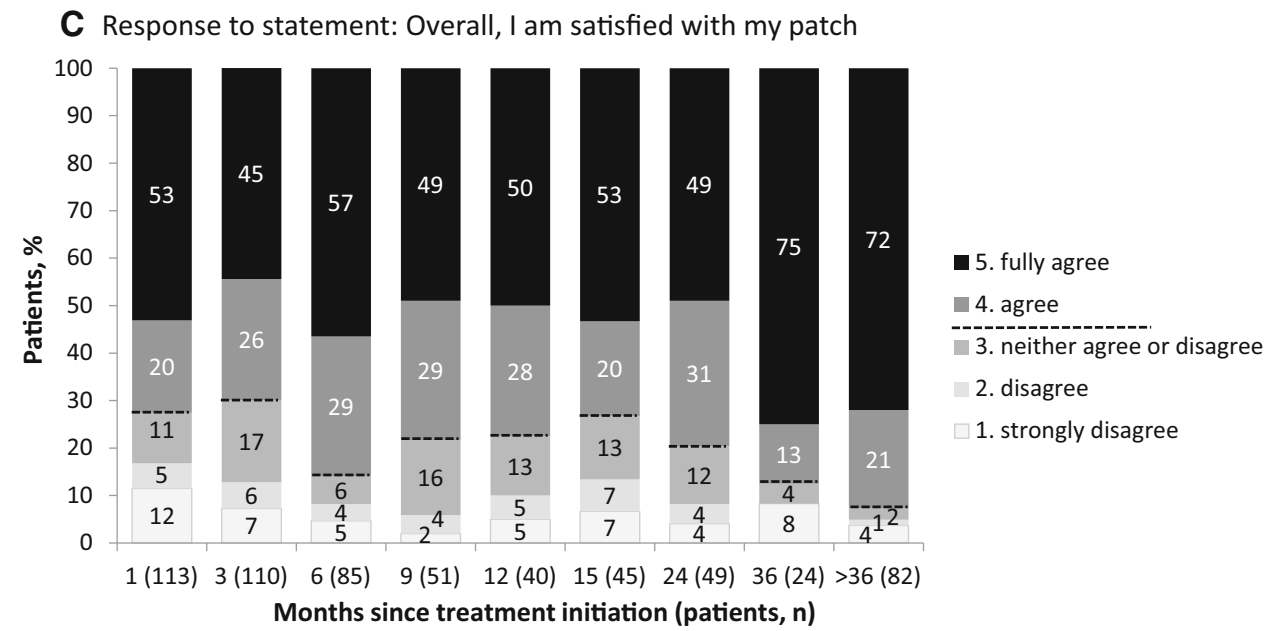

constipation at study baseline, including $21.9 \%$ of TDBnaïve patients, is worth noting. While constipation is a common class effect of opioid analgesia, arising from the interaction of opioids with $\mu$-opioid receptors present throughout the gastrointestinal tract, other factors may also contribute to constipation-for example, reduced mobility and dietary factors $[36,37]$. Furthermore, some commonly used drugs-for example, selective serotonin reuptake inhibitors-are also associated with an increased incidence of constipation [38]. 
Skin irritancy appears to be a class effect for transdermal delivery of opioid. A systematic review including five studies of transdermal fentanyl identified skin irritation at the application site to be the only AE that was not observed in patients receiving opioid analgesia via other modes of delivery [39]. Skin irritations reported in the present study were generally mild or moderate in severity and were restricted to the application site. Furthermore, very few patients discontinued TDB therapy because of this AE. However, only one quarter of patients experiencing skin irritations received treatment to manage these symptoms. Given that most patients reported that the interventions were at least somewhat effective, physicians are likely missing opportunities to manage skin irritations associated with TDB. It is the authors' clinical experience that advising patients to wash the affected area with soap and water after removing the TDB patch can help to ameliorate minor skin irritations, along with application of a low-dose steroid cream for a few days if symptoms persist. Another observation from this study, which may assist clinicians to optimize TDB therapy, was that skin irritations tended to be more common in patients with a history of skin reactions to certain foods, perfumes, cosmetics, and washing powder. Proactive questioning about skin irritations and their management appears to be particularly relevant for these patients. Furthermore, the higher incidence rates of constipation and skin irritations reported in TDB-experienced patients highlight the need for physicians to proactively address these common AEs, particularly in individuals receiving long-term therapy. It is possible that the $\leq 9$-month duration of treatment for treatment-naïve patients may not been of a sufficient duration for some AEs to develop.

In comparison with a prior 12-month, retrospective cohort study of nearly 5000 UK patients prescribed lowdose TDB by primary care physicians, the incidences of constipation, nausea, vomiting, and dizziness were largely similar, while skin irritations were 5- to 30-fold more frequent in the present study [40]. The reason for this discrepancy is unclear, although it may reflect the different designs of the studies. For example, the larger study utilized information obtained from the General Practice Research Database, while data were obtained from studyspecific questionnaires completed at regular intervals during the present study and included some patients who were already receiving 7-day TDB at study entry [40].

Despite the occurrence of AEs, discontinuation of TDB therapy was rare. Over $80 \%$ of patients continued treatment throughout the follow-up period, with just $12.0 \%$ discontinuing because of AEs. Discontinuation was also uncommon in patients who experienced skin irritations. This suggests that the benefits of TDB outweigh the AEs, which patients generally tolerate. While direct comparisons between studies of differing designs cannot be made, the rate of treatment discontinuation due to AEs was lower than those reported in randomized, controlled trials of lowdose TDB (approximately $36 \%$ ), was similar to those observed in studies of transdermal fentanyl (approximately $12 \%$ ), and compares favorably with those in studies of oral opioid analgesics (approximately $23 \%$ ) [30, 32, 39]. Other studies of low-dose TDB have also demonstrated high rates of treatment continuation, which were significantly greater than those observed with codeine, dihydrocodeine, and tramadol [40].

This study was designed to capture both patients' and physicians' perceptions of treatment with TDB. Approximately one fifth of skin irritation events reported by physicians were not also reported by patients. While this discrepancy between patient- and physician-reported AEs may be due to the design of this study, it underscores the importance of discussing AEs during consultations. It is also worth noting that the TDB patch strength tended to increase over the course of treatment, suggesting that some physicians do not reduce the buprenorphine dosage prior to discontinuation in order to ameliorate AEs.

This study also indicates that in real-world settings, most patients use 7-day TDB patches in accordance with treatment recommendations. Most patients wore each patch for the recommended duration and were rotating the skin site for patch application. Very few patients forgot to apply the patch, applied more patches than were prescribed, cut the patch, or applied a new patch before the previous one was removed. The high rate of self-reported adherence to treatment in this study is an important observation, given that patients who are prescribed self-administered medications typically take only about half of their prescribed doses, and few data are available to provide insight into how adherence can be improved to realize the full health benefits of medicines [41-43]. The high rates of self-reported adherence tallied with the observation that most patients were 'satisfied' or 'very satisfied' with TDB therapy, including those who experienced skin irritations. Treatment satisfaction rates appeared to increase over time, with $92.7 \%$ of patients who used the patch for $\geq 36$ months reporting high satisfaction.

The advantages of transdermal opioids include slow, continuous release into the circulation over a prolonged period and the avoidance of first-pass hepatic metabolism [15]. Low-dose TDB also has the convenience of onceweekly administration and reduces the overall pill burden, which may be particularly important for older patients with chronic conditions who are commonly taking multiple medications [32]. However, patch therapy is associated with some limitations, including less flexible dosage adjustment in comparison with oral opioid formulations [10]. 
While this study was designed to inform on the effectiveness, AE profile, self-reported adherence, and perceptions of treatment with TDB in routine clinical practice, because of the observational, non-intervention design, the efficacy of TDB cannot be compared with that of other treatments. Other limitations included potential patient selection bias and lack of validation of outcome measures.

\section{Conclusion}

This UK observational study indicated that in everyday clinical practice, TDB was well tolerated by patients with a variety of chronic pain conditions and comorbidities. Most patients reported that TDB therapy was effective and were satisfied with their treatment. Self-reported adherence to TDB was also very high, with nearly all patients applying the patches per treatment recommendations. Although many patients receiving TDB experienced at least one $\mathrm{AE}$, these were tolerable, as AEs rarely resulted in treatment discontinuation. This study also identified potential missed opportunities to ameliorate or reduce the intensity of common AEs experienced by patients treated with TDB.

Acknowledgments Medical writing support was provided by Siân Marshall, PhD, of Siantifix Ltd, Cambridge, UK. The authors would like to thank all of the investigators and study participants.

\section{Compliance with Ethical Standards}

All authors contributed to the analysis and/or interpretation of data, as well as critical revision of the manuscript for important intellectual content, and approved the submitted draft.

All study documents were reviewed and approved by the appointed Research Ethics Committee, and all patients participating in this study provided informed, written consent.

Funding The study was funded by Mundipharma Research $\mathrm{GmbH}$ \& Co. KG. Medical writing support was funded by Mundipharma Research GmbH \& Co. KG.

Potential conflicts of interest Mick Serpell was the Principal Investigator for the study. He received support from Mundipharma Research $\mathrm{GmbH} \& \mathrm{Co} . \mathrm{KG}$ to travel to meetings to discuss the findings of this study and discuss manuscript preparation. He has also received honoraria from Astellas, Grünenthal Ltd, Sanofi-Pasteur MSD, Napp Pharmaceuticals and Pfizer to present at educational meetings. Sabine Scherzinger and Shiva Tripathi acted as study investigators. Shiva Tripathi has received honoraria from Mundipharma Research GmbH \& Co. KG, Napp Pharmaceuticals, Astellas and Pfizer for speaking at scientific meetings. Sabine Scherzinger has no potential conflicts of interest to declare. Sònia Rojas-Farreras, an employee of IMS Health at the time of the study, was responsible for statistical analysis of the data and preparation of the study report, funded by Mundipharma Research $\mathrm{GmbH} \&$ Co. KG. Alexander Oksche, an employee of Mundipharma Research GmbH \& Co. KG, acted as the responsible clinician for the study sponsor and contributed to the design of the study. Margaret Wilson, an employee of Mundipharma Research Ltd, also contributed to the design of the study. As corresponding author, Margaret Wilson acts as the guarantor of the manuscript.

Statement of human rights All procedures performed in this study involving human participants were in accordance with the ethical standards of the institutional research ethics committees and with the 1964 Declaration of Helsinki and its later amendments or comparable ethical standards.

Open Access This article is distributed under the terms of the Creative Commons Attribution-NonCommercial 4.0 International License (http://creativecommons.org/licenses/by-nc/4.0/), which permits any noncommercial use, distribution, and reproduction in any medium, provided you give appropriate credit to the original author(s) and the source, provide a link to the Creative Commons license, and indicate if changes were made.

\section{References}

1. Breivik H, Collett B, Ventafridda V, et al. Survey of chronic pain in Europe: prevalence, impact on daily life, and treatment. Eur J Pain. 2006;10:287-333.

2. Helme RD, Gibson SJ. The epidemiology of pain in elderly people. Clin Geriatr Med. 2001;17:417-31.

3. Strine TW, Hootman JM, Chapman DP, et al. Health-related quality of life, health risk behaviors, and disability among adults with pain-related activity difficulty. Am J Public Health. 2005;95:2042-8.

4. Allen H, Hubbard D, Sullivan S. The burden of pain on employee health and productivity at a major provider of business services. J Occup Environ Med. 2005;47:658-70.

5. Vargas-Schaffer G. Is the WHO analgesic ladder still valid? Twenty-four years of experience. Can Fam Physician. 2010;56:514-5.

6. Machado GC, Maher CG, Ferreira PH, et al. Efficacy and safety of paracetamol for spinal pain and osteoarthritis: systematic review and meta-analysis of randomised placebo controlled trials. BMJ. 2015;350:h1225.

7. Chou R, Fanciullo GJ, Fine PG, et al. Clinical guidelines for the use of chronic opioid therapy in chronic noncancer pain. J Pain. 2009;10:113-30.

8. Pergolizzi J, Boger RH, Budd K, et al. Opioids and the management of chronic severe pain in the elderly: consensus statement of an international expert panel with focus on the six clinically most often used World Health Organization step III opioids (buprenorphine, fentanyl, hydromorphone, methadone, morphine, oxycodone). Pain Pract. 2008;8:287-313.

9. McCracken LM, Velleman SC, Eccleston C. Patterns of prescription and concern about opioid analgesics for chronic nonmalignant pain in general practice. Prim Healthc Res Dev. 2008;9:146-56.

10. Plosker GL, Lyseng-Williamson KA. Buprenorphine 5, 10 and 20 mug/h transdermal patch: a guide to its use in chronic non-malignant pain. CNS Drugs. 2012;26:367-73.

11. Hadley G, Derry S, Moore RA, et al. Transdermal fentanyl for cancer pain. Cochrane Database Syst Rev. 2013;(10):CD010270.

12. Livingston KE, Traynor JR. Disruption of the Na+ ion binding site as a mechanism for positive allosteric modulation of the muopioid receptor. Proc Natl Acad Sci USA. 2014;111:18369-74.

13. Greenwald MK, Johanson CE, Moody DE, et al. Effects of buprenorphine maintenance dose on mu-opioid receptor availability, plasma concentrations, and antagonist blockade in heroin- 
dependent volunteers. Neuropsychopharmacology. 2003;28:2000-9.

14. Zubieta J, Greenwald MK, Lombardi U, et al. Buprenorphineinduced changes in mu-opioid receptor availability in male heroin-dependent volunteers: a preliminary study. Neuropsychopharmacology. 2000;23:326-34.

15. Kress HG. Clinical update on the pharmacology, efficacy and safety of transdermal buprenorphine. Eur J Pain. 2009;13:219-30.

16. Pergolizzi J, Aloisi AM, Dahan A, et al. Current knowledge of buprenorphine and its unique pharmacological profile. Pain Pract. 2010;10:428-50.

17. Guetti C, Angeletti C, Marinangeli F, et al. Transdermal buprenorphine for central neuropathic pain: clinical reports. Pain Pract. 2011;11:446-52.

18. Aurilio C, Ceccarelli I, Pota V, et al. Endocrine and behavioural effects of transdermal buprenorphine in pain-suffering women of different reproductive ages. Endocr J. 2011;58:1071-8.

19. Pergolizzi JV Jr, Scholten W, Smith KJ, et al. The unique role of transdermal buprenorphine in the global chronic pain epidemic. Acta Anaesthesiol Taiwan. 2015;53:71-6.

20. Napp Pharmaceuticals Limited. Transtec: summary of product characteristics. Cambridge: Napp Pharmaceuticals Limited; 2014.

21. Napp Pharmaceuticals Limited. BuTrans: summary of product characteristics. Cambridge: Napp Pharmaceuticals Limited; 2014.

22. De Barutell C, Gonzalez-Escalada JR. Efficacy and safety of buprenorphine TDS in conjunction with oral tramadol or morphine as rescue medication in the treatment of 390 patients with chronic pain: a summary of two retrospective Spanish multicenter studies. J Appl Ther Res. 2007;5:14-24.

23. Zarth R. Comparison of buprenorphine, morphine sulphate and fentanyl citrate as rescue medication for breakthrough pain in cancer patients. J Appl Ther Res. 2008;6:15-9.

24. Zin CS, Chen LC, Knaggs RD. Changes in trends and pattern of strong opioid prescribing in primary care. Eur $\mathrm{J}$ Pain. 2014;18:1343-51.

25. Breivik H, Marte Ljossa T, Stengaard-Pedersen K, et al. A 6-months, randomised, placebo-controlled evaluation of efficacy and tolerability of a low-dose 7-day buprenorphine transdermal patch in osteoarthritis patient naive to potent opioids. Scand J Pain. 2010;1:122-41.

26. Gordon A, Callaghan D, Spink D, et al. Buprenorphine transdermal system in adults with chronic low back pain: a randomized, double-blind, placebo-controlled crossover study, followed by an open-label extension phase. Clin Ther. 2010;32:844-60.

27. Landau CJ, Carr WD, Razzetti AJ, et al. Buprenorphine transdermal delivery system in adults with persistent noncancer-related pain syndromes who require opioid therapy: a multicenter, 5-week run-in and randomized, double-blind maintenance-ofanalgesia study. Clin Ther. 2007;29:2179-93.

28. Steiner DJ, Sitar S, Wen W, et al. Efficacy and safety of the seven-day buprenorphine transdermal system in opioid-naive patients with moderate to severe chronic low back pain: an enriched, randomized, double-blind, placebo-controlled study. J Pain Symptom Manage. 2011;42:903-17.

29. Poulain P, Denier W, Douma J, et al. Efficacy and safety of transdermal buprenorphine: a randomized, placebo-controlled trial in 289 patients with severe cancer pain. J Pain Symptom Manage. 2008;36:117-25.

30. James IG, O'Brien CM, McDonald CJ. A randomized, doubleblind, double-dummy comparison of the efficacy and tolerability of low-dose transdermal buprenorphine (BuTrans seven-day patches) with buprenorphine sublingual tablets (Temgesic) in patients with osteoarthritis pain. J Pain Symptom Manage. 2010;40:266-78.

31. Karlsson M, Berggren AC. Efficacy and safety of low-dose transdermal buprenorphine patches $(5,10$, and $20 \mu \mathrm{g} / \mathrm{h})$ versus prolonged-release tramadol tablets $(75,100,150$, and $200 \mathrm{mg}$ ) in patients with chronic osteoarthritis pain: a 12-week, randomized, open-label, controlled, parallel-group noninferiority study. Clin Ther. 2009;31:503-13.

32. Conaghan PG, O'Brien CM, Wilson M, et al. Transdermal buprenorphine plus oral paracetamol vs an oral codeine-paracetamol combination for osteoarthritis of hip and/or knee: a randomised trial. Osteoarthritis Cartilage. 2011;19:930-8.

33. Vander HK, Parera AE, Jacobs C, et al. Allergic contact dermatitis from transdermal buprenorphine. Contact Dermatitis. 2008;59:366-9.

34. Graziottin A, Gardner-Nix J, Stumpf M, et al. Opioids: how to improve compliance and adherence. Pain Pract. 2011;11:574-81.

35. Elliott AM, Smith BH, Penny KI, et al. The epidemiology of chronic pain in the community. Lancet. 1999;354:1248-52.

36. Muller-Lissner S. Opioid-induced constipation-mechanisms, relevance and management. Eur Gastroenterol Hepatol Rev. 2010;6:54-7.

37. Holzer P, Ahmedzai SH, Niederle N, et al. Opioid-induced bowel dysfunction in cancer-related pain: causes, consequences, and a novel approach for its management. J Opioid Manag. 2009;5:145-51.

38. Marks DM, Park MH, Ham BJ, et al. Paroxetine: safety and tolerability issues. Expert Opin Drug Saf. 2008;7:783-94.

39. Noble M, Treadwell JR, Tregear SJ, et al. Long-term opioid management for chronic noncancer pain. Cochrane Database Syst Rev. 2010;(1):CD006605.

40. Gallagher AM, Leighton-Scott J, van Staa TP. Utilization characteristics and treatment persistence in patients prescribed low-dose buprenorphine patches in primary care in the United Kingdom: a retrospective cohort study. Clin Ther. 2009;31:1707-15.

41. Alhewiti A. Adherence to long-term therapies and beliefs about medications. Int J Family Med. 2014;2014:479596.

42. Nieuwlaat R, Wilczynski N, Navarro T, et al. Interventions for enhancing medication adherence. Cochrane Database Syst Rev. 2014;(11):CD000011.

43. World Health Organization (WHO). Adherence to long-term therapies: evidence for action. Geneva: WHO; 2015. 